\title{
AMPHISTOMES (TRÉMATODES) DES RUMINANTS DOMESTIQUES DE LA RÉPUBLIQUE DU TCHAD DESCRIPTION D'UN GASTROTHYLACIDAE NOUVEAU CARMYERIUS GRABERI n. sp.
}

\author{
Par Simon GRETILlat
}

A la fin de 1959, le $\mathrm{D}^{\mathrm{r}}$ Graber, Chef du Service de Parasitologie au Laboratoire de l'Elevage de Farcha, Fort-Lamy (République du Tchad), nous envoyait pour étude et détermination un lot d'Amphistomata Rud. 1801, recueillis par ses soins sur des bovins, ovins et caprins aux abattoirs de Fort-Lamy, Ati, Bongor et Abecher.

Nous donnons ci-dessous les résultats de l'étude de ce matériel.

\section{A) PARAMPHistomatidE Travassos, 1934.}

I. Paramphistominæ L. Travassos, 1934.

Paramphistomum microbothrium Fischoeder, 1901.

Chez Bos taurus L. à Fort-Lamy, Ati et Bongor.

Chez Ovis aries L. à Ati, Bongor et Abecher.

Chez Capra hircus L. à Ati et Abecher.

Cette espèce semble très répandue en Afrique Equatoriale, tant chez les Bovins que chez les Ovins et Caprins.

Calicophoron calicophorum (Fischoeder, 1901) Näsmark, 1937.

Dans le rumen de Bos taurus à Fort-Lamy et Bongor, et chez Ovis aries à Fort-Lamy et Ati.

Cette espèce paraît être moins fréquente que la précédente.

Bothriophoron bothriophoron (M. Bräun, 1892) Stiles et Goldberger, $1910=$ Paramphistomum bothriophoron (M. Bräun, 1892) Fischoeder, 1901.

Une vingtaine de spécimens chez Bos taurus à Fort-Lamy. Il est intéressant de signaler que cette espèce, qui est très fréquente dans les régions Nord-Ouest de Madagascar, n'avait été trouvée en Afrique qu'une seule fois par le $\mathrm{D}^{r}$ Dinnik, de l'East African Veterinary Research Organization de Muguga, Nairobi (Kénya), en 1950, aux 
abattoirs de Nairobi, dans un mouton et une chèvre (Rapport de l'East African Research Organization, 1952). Lors de notre étude sur le genre Bothriophoron Stiles et Goldberger, 1910, publiée en 1958 , le $\mathrm{D}^{\mathrm{r}}$ Dinnik nous avait fait part de cette découverte et communiqué des coupes histologiques de ses spécimens. Depuis lors, cet auteur n'a jamais pu retrouver d'exemplaires de cette espèce décrite de Madagascar et qui existe aussi en Afrique Equatoriale.

\section{Stephanopharynginæ Stiles et Goldberger, 1910.}

Stephanopharynx compactus Fischoeder, 1901.

Les exemplaires que nous avons étudiés proviennent de la panse de deux bœufs sacrifiés aux abattoirs de Fort-Lamy. Nous avons tout d'abord hésité à les considérer comme des $S$. compactus. En effet, nos spécimens présentent des caractères (acétabulum, diverticule pharyngien, glandes vitellogènes) qui sont intermédiaires entre ceux de cette espèce et ceux de Stephanopharynx secundus Stunkard, 1929. Cependant, après avoir consulté les travaux de Maplestone (1923) et Näsmark (1937), nous pensons pouvoir rattacher notre matériel à l'espèce de Fischoeder.

B) GASTROTHYLACIDE Stiles et Goldberger, 1910.

I) Carmyerius spatiosus (Brandes, 1898).

Dans la panse de Bos taurus et Ovis aries à Fort-Lamy.

II) Carmyerius graberi n. sp.

Le matériel d'après lequel nous faisons l'étude descriptive de cette espèce comporte 18 spécimens récoltés dans le rumen d'un bouvillon et 7 exemplaires provenant de la panse d'un mouton, tous les deux sacrifiés aux abattoirs de Fort-Lamy (République du Tchad), le premier le 5 septembre 1959 et le second en septembre 1954.

Pour l'étude anatomique des organes internes, nous avons eu recours à la technique des coupes histologiques sériées et colorées à l'hémalun-éosine. Dix exemplaires ont été coupés longitudinalement et cinq transversalement. Les mensurations et les positions relatives des différents organes internes que nous donnons au cours de cette description correspondent à des valeurs moyennes.

Dimensions et habitus (fig. $\mathrm{n}^{\circ \times} 1$ et 2 ) :

Corps long de 3 à $5 \mathrm{~mm}$. et large de 3 à $4 \mathrm{~mm}$. Coupe transversale sensiblement circulaire. La partie antérieure présente l'orifice de la poche centrale, la bouche étant subterminale et en position légère- 
ment dorsale. La partie postérieure du Trématode est occupée par un acétabulum à cavité très peu profonde, à bords légèrement saillants, et de contour circulaire.

Le corps présente latéralement et un peu au-dessus de la ventouse postérieure. deux renflements qui correspondent aux deux masses testiculaires. Les exemplaires en état de rétraction au moment de leur mort sont presque sphériques (habitus d'un pois).

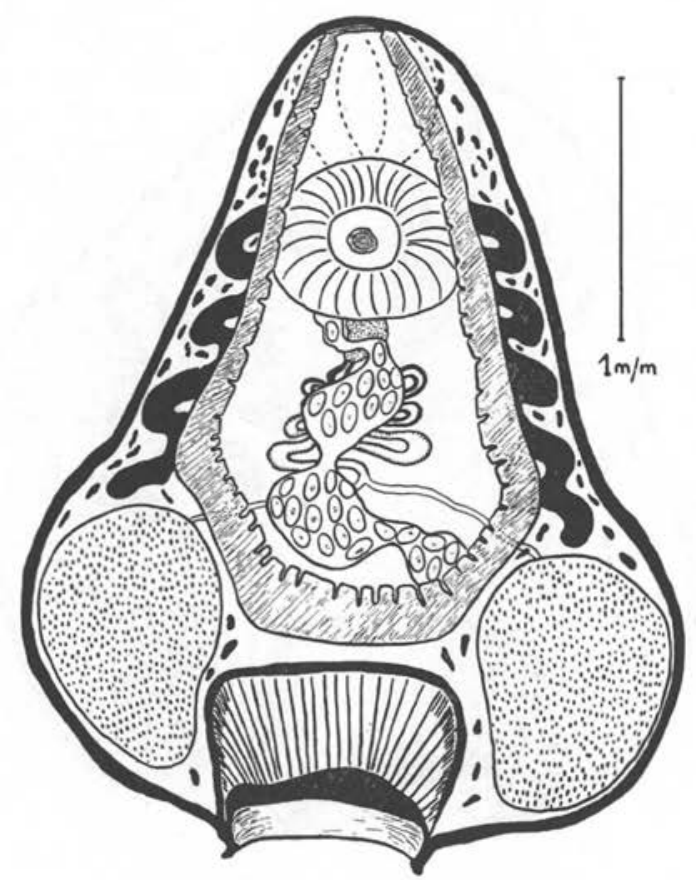

FIG. 1. - Carmyerius graberi n. sp., coupe schématique vue de face.

\section{Guticule externe :}

Son épaisseur qui est maximum dans la partie postérieure du ver atteint $70 \mu$. Après coloration à l'hémalun-éosine, elle parait formée de deux couches : l'une externe, mince, qui se colore en violet clair, et l'autre interne, plus épaisse, colorée en rose.

\section{Tube digestif :}

La bouche subterminale est située à environ $360 \mu$ de l'orifice de la poche centrale. Elle se trouve au fond d'un léger renfoncement cuticulaire. 
La ventouse buccale, subsphérique, a un diamètre de $500 \mu$. Sa paroi, fortement musclée, a une épaisseur de 170 à $200 \mu$. Sa partie postérieure est accolée à la masse musculaire du pore génital.

Un œsophage très court ( 300 à $320 \mu$ ) fait suite sans transition à la ventouse orale, dont il suit la face postérieure en se dirigeant vers la face dorsale du ver. Ses parois sont musculeuses et épaisses de 20 à $40 \mu$. Il se termine par la bifurcation cæcale située au niveau du pore génital.

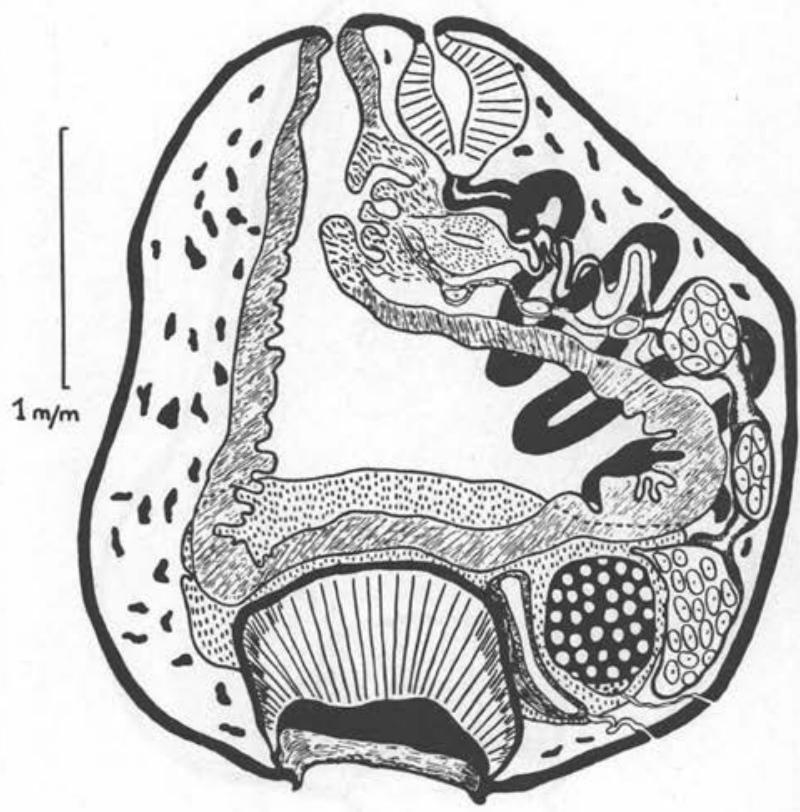

Fig. 2. - Carmyerius graberi n. sp., coupe schématique dorso-ventrale.

Les deux cæca, dès leur origine, se dirigent, chacun, vers les parties latérales du corps, pour se terminer aux environs du pôle supérieur de chaque testicule, après avoir décrit de nombreuses anses Leur diamètre interne est de $80 \mu$ environ.

\section{Poche centrale (fig. $\mathrm{n}^{\circ *} 1,2,3$ ) :}

Elle occupe environ le tiers du volume du Trématode et s'ouvre par un orifice en forme de fente transversale dirigée perpendiculairement à l'axe sagittal de l'helminthe. Ses parois sont très épaisses, 100 à $200 \mu$, et colorées en rose orangé par l'hémalun-éosine. 
La poche centrale a une section transversale à peu près circulaire au niveau de la partie moyenne du corps. Elle présente une portion antérieure étroite et aplatie dorso-ventralement, où se trouve le pore génital, et une portion postérieure spacieuse, s'appuyant sur le plafond de la ventouse postérieure, les faces latérales des testicules, l'ovaire et le gros peloton utérin placé immédiatement en arrière des glandes femelles. Dans sa partie postéro-dorsale, la

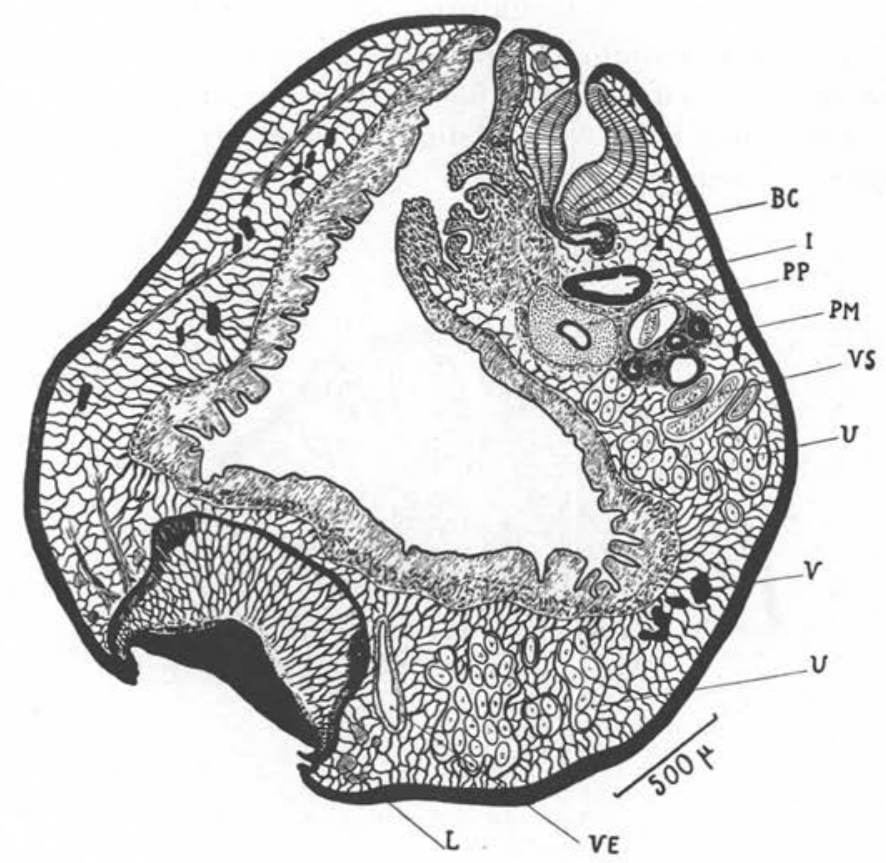

FIG. 3. - Carmyerius graberi, coupe histologique longitudinale passant par le pore génital.

poche centrale se rapproche de la cuticule du ver, jusqu'à environ $200 \mu$. Par contre, ventralement, la paroi de ce diverticule est parallèle à la face externe du Trématode et en est distante de 400 à $500 \mu$.

Le pore génital s'ouvre dans la poche ventrale à environ $750 \mu$ de son orifice. En arrière et au-dessous du pore génital, la paroi du corps est très épaisse, 1.000 à $1.200 \mu$, et renferme la musculature très développée de la papille génitale, la pars prostatica, la pars musculosa, la vésicule séminale et les dernières anses utérines. 
Acétabulum (fig. $\mathrm{n}^{\circ} 7$ ) :

Il a, dans l'espèce qui nous intéresse, une constitution tout à fait spéciale. Sur les 15 spécimens étudiés en coupes histologiques et sur les 10 exemplaires conservés dans l'alcool, l'acétabulum présente les caractères suivants :

Ventouse postérieure en position terminale, à contour externe sensiblement circulaire, à bords légèrement saillants, d'un diamètre d'environ $1 \mathrm{~mm}$. et très peu profonde : 120 à $130 \mu$.

Sur coupes histologiques, la paroi de cet organe a dans sa partie centrale une épaisseur de 500 à $550 \mu$, alors que latéralement elle ne mesure que 100 à $125 \mu$. L'acétabulum peut être comparé à une demi-sphère à peu près pleine.

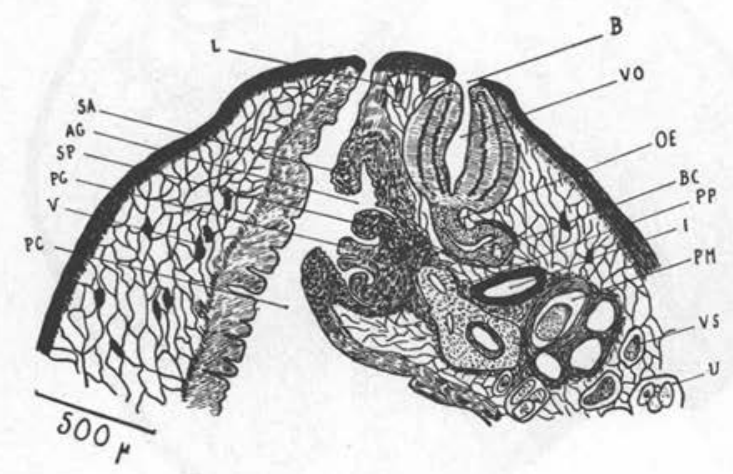

FIG. 4. - C. graberi, coupe histologique longitudinale. Pore génital et ventouse orale.

Le plancher, ou face inférieure de la ventouse, est formé par une paroi cuticulaire d'environ 20 à $25 \mu$ d'épaisseur et par une musculeuse à fibres très denses, de 80 à $130 \mu$ de profondeur.

Le plafond de la ventouse est faiblement musculeux dans sa partie centrale ; par contre, dans ses parties latérales, et jusqu'au niveau des bords de l'acétabulum, sa paroi est doublée de très forts et très nombreux faisceaux musculaires, formant une musculeuse dont l'épaisseur varie de 50 à $120 \mu$.

La partie centrale de la paroi de l'acétabulum est occupée par un tissu fibro-musculaire plus ou moins lâche, qui relie le plancher au plafond de l'organe. 
Appareil génital :

PoRe génital (fig. $n^{\circ} 4$ ) : Il est particulièrement bien développé puisque son diamètre, $600 \mu$, sphincter atrial compris, est plus grand que celui de la ventouse orale.

Comme chez Carmyerius synethes (Fischoeder, 1901) et che\% Bothriophoron bothriophoron (M. Bräun, 1892) Stiles et Goldberger, 1910, la papille génitale est placée au fond d'un atrium génital bien développé.

Cet atrium, dont la profondeur est de $300 \mu$ et le diamètre de $450 \mu$ environ, est circonscrit par un sphincter puissant, dont l'épaisseur varie de 150 à $200 \mu$, et qui est formé par une densification des fibres musculaires de la paroi de la poche centrale. Ces fibres entourent tout l'atrium et sont plus denses au niveau de son orifice.

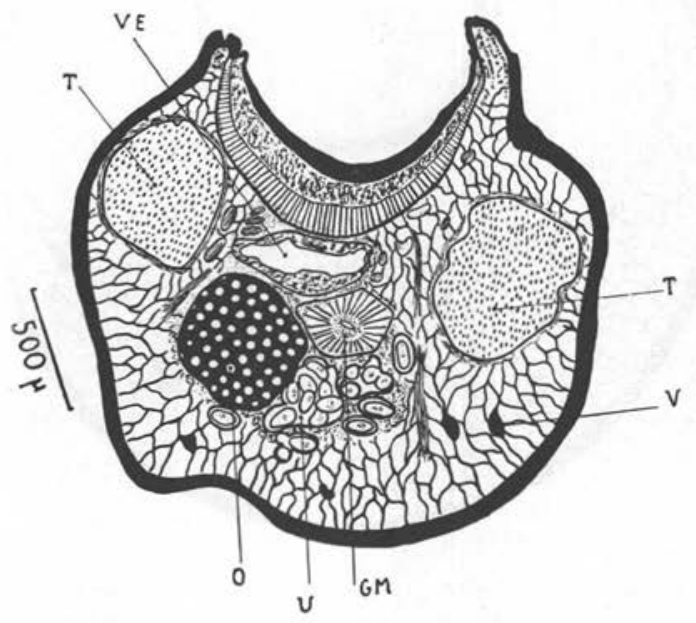

Fig. 5. - C. graberi, coupe histologique transversale passant au niveau de l'ovaire et de la glande de Mehlis.

Au fond de la cavité ainsi formée, est placée la papille génitale entourée elle-même d'un sphincter papillaire de $375 \mu$ de diamètre. La papille, qui a sensiblement la forme d'un chapeau de champignon, a $150 \mu$ de long, et son extrémité a $120 \mu$ de diamètre. A son sommet, débouche le canal hermaphrodite, de 150 à $170 \mu$ de longueur.

Appareil génital mâle (fig. $\mathrm{n}^{\circ *} 1,2,5$ et 6 ) : Les deux testicules sont directement appliqués contre la cuticule externe du ver et for- 
ment, à l'extérieur, deux protubérances arrondies de chaque côté de l'acétabulum.

Pratiquement sans lobulation apparente, ils ont une forme allongée et s'étendent d'avant en arrière, entre le fond de la poche centrale, les parois latérales de l'acétabulum, et la paroi externe du corps du Trématode. Leur masse ne repousse pas, vers le haut, le fond de la poche centrale.

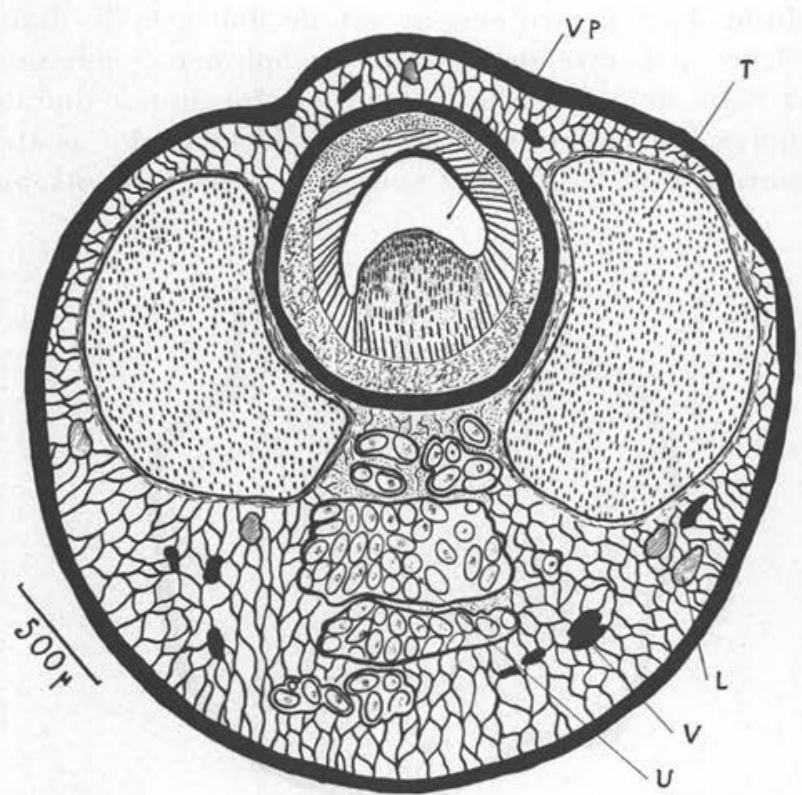

FIG. 6. - C. graberi, coupe histologique transversale passant au niveau de la partie moyenne des testicules.

Ils sont volumineux et leurs dimensions sont les suivantes :

— diamètre antéro-postérieur : 1.500 à $1.700 \mu$;

— diamètre transversal : 750 à $900 \mu$.

Les canaux déférents débutent dans la partie moyenne de la face interne des testicules. Ils se dirigent vers la région dorsale du ver pour s'unir en un déférent unique à la limite du tiers postérieur et du tiers moyen du corps. Ce canal remonte en position dorsale aux côtés des anses utérines. Au niveau de la limite, tiers antérieur-tiers moyen du Trématode, il se renfle en une vésicule séminale courte, 
contournée sur elle-même, qui se poursuit en avant par une pars musculosa courte, dont les anses forment un glomérule de 375 à $400 \mu$ de diamètre. Histologiquement, la pars musculosa est formée d'une tunique externe épaisse de 10 à $12 \mu$, à fibres longitudinales, et d'une tunique interne dont l'épaisseur varie de 25 à $35 \mu$, constituée par des fibres transversales. Elle a son calibre interne maximum dans sa partie distale : 120 à $130 \mu$.

La pars prostatica fait immédiatement suite à cette partie musculeuse. Elle est épaisse, trapue, et a une longueur de 500 à $600 \mu$.

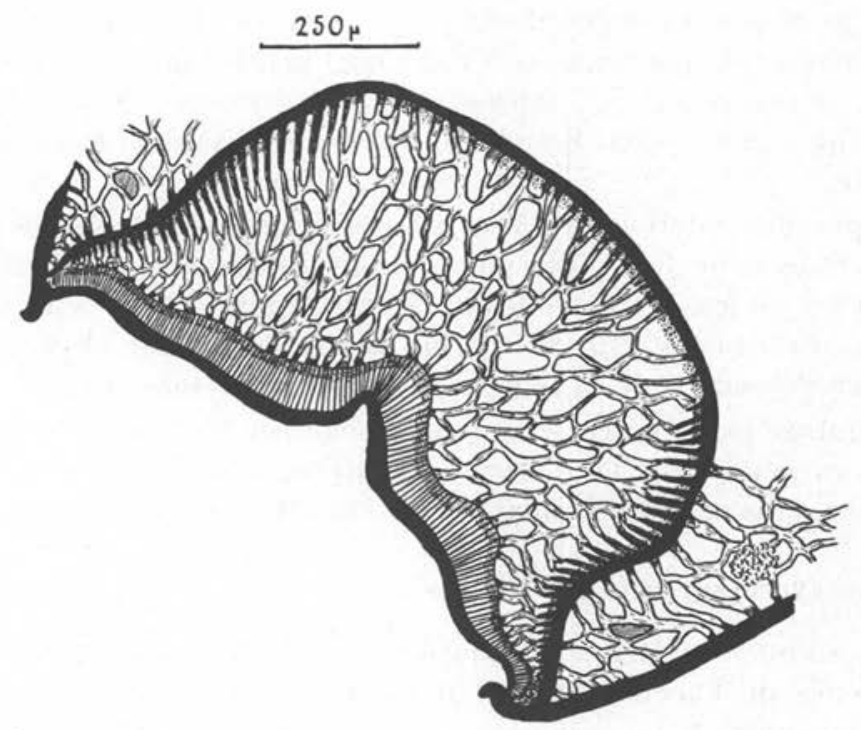

FIG. 7. - C. graberi, coupe histologique longitudinale de la ventouse postérieure.

Antérieurement, elle débouche dans le canal éjaculateur qui s'unit au canal femelle pour former le canal hermaphrodite s'ouvrant au sommet de la papille génitale. Ces trois derniers canaux sont noyés dans la masse musculaire du pore génital.

APPareil GÉNital FEMELle (fig. $\mathrm{n}^{\circ s} 1,2,4$ et 5) : L'ovaire est subsphérique et a un diamètre de 450 à $500 \mu$. Il est placé entre les deux testicules, immédiatement sous le fond de la poche centrale, en arrière de la vésicule excrétrice et de l'acétabulum, en position très postérieure et submédiane.

La glande de Mehlis lui est contiguë, mais un peu plus postérieure. Son diamètre antéro-postérieur est de $300 \mu$ et son diamètre transversal de $250 \mu$. 
Le canal de Laurer est court et, après 2 ou 3 sinuosités, débouche sur la face dorsale du ver, au niveau de son cinquième postérieur.

En arrière et au-dessous de l'ovaire et de la glande de Mehlis, l'utérus se renfle en un diverticulum seminis uterinum, où se trouvent, parmi les nombreux œufs qu'il renferme, des amas de spermatozoïdes.

Après ce diverticule, l'utérus se dirige vers la paroi dorsale du ver et remonte vers le pore génital en décrivant des anses plus ou moins renflées dans la région médio-dorsale de l'helminthe, puis il s'infléchit vers l'avant pour passer sous la vésicule séminale, entre celle-ci et la paroi dorsale de la poche centrale. Il occupe alors une position ventrale par rapport à l'appareil génital mâle (pars musculosa et pars prostatica). Après quelques circonvolutions, l'utérus aboutit au canal génital femelle, qui débouche dans le canal hermaphrodite.

Les glandes vitellogènes sont groupées en amas plus ou moins lâches. Elles sont disséminées dans le tissu aréolaire de soutien qui remplit les interstices entre les différents organes. Elles sont uniformément distribuées dans les parois ventrale, latérale et dorsale du corps, et s'étendent de la bouche à la région acétabulaire.

Les quinze exemplaires examinés en coupes histologiques présentaient des œufs dans leur utérus. In utero, nous leur avons trouvé les dimensions suivantes : longueur : 125 à $130 \mu$, largeur : 70 à $75 \mu$.

\section{Système excréteur (fig. $\mathrm{n}^{\circ \times} 2$ et 4 ) :}

La vésicule excrétrice est placée en arrière de la paroi latéropostérieure de l'acétabulum. Latéralement, elle est contiguë aux deux testicules et, postérieurement, elle s'appuie sur l'ovaire et la glande de Mehlis. En position antérieure, elle atteint le plancher de la poche centrale, mais n'envoie pas de diverticule entre celui-ci et le plafond de la ventouse postérieure. Le canal excréteur est court, très peu contourné et se termine au niveau du pore excréteur situé un peu plus en arrière que l'orifice du canal de Laurer sur la face dorsale du corps du Trématode.

\section{Système lymphatique :}

Il semble constitué par deux troncs longitudinaux qui envoient des ramifications secondaires vers les différents organes, et particulièrement dans les régions orale et acétabulaire. 


\section{Discussion}

Parmi les Amphistomata, la famille des Gastrothylacidæ Stiles et Goldberger 1910 est caractérisée par l'existence d'une poche centrale. D'après l'ouvrage de Skrjabin (1949), elle comprend trois genres : Gastrothylax Poirier, 1883, Fischoederius Stiles et Goldberger, 1910 et Carmyerius Stiles et Goldberger, 1910.

Si nous adoptons la classification proposée par Stiles et Goldberger en 1910, l'espèce que nous venons de décrire appartient au genre Carmyerius (testicules postérieurs placés sensiblement côte à côte et séparés par un espace médian où sont placés l'ovaire, la glande de Mehlis et le début de l'utérus qui remonte vers la partie antérieure de l'helminthe en position dorso-médiane).

A l'heure actuelle, le genre Carmyerius comprend les onze espèces suivantes :

Carmyerius gregarius (Loos, 1896), espèce-type, trouvée dans l'estomac de Bos bubalis L. en Egypte.

Carmyerius spatiosus (G. Brandes, 1898) chez Bos taurus L. en Arabie.

Carmyerius synethes (Fischoeder, 1901) trouvé dans la panse d'un Bos bubalis sondaicus (Rutimeyer) (Bos kerabau) mort au parc zoologique de Berlin et provenant de Ceylan.

Carmyerius minutus (Fischoeder, 1901) dans l'estomac de l'antilope Tragelaphus scriptus Pallas [Yaoundé (Cameroun)].

Carmyerius mancupatus (Fischoeder, 1902) dans le rumen de Bos taurus L. (Afrique).

Carmyerius wenyoni (Leiper, 1908) trouvé dans l'estomac de Cobus maria Gray, à Taufikia (Nil Blanc).

Carmyerius cruciformis (Leiper, 1910) dans l'estomac d'un hippopotame tué sur les bords du lac Victoria (Nyanza).

Carmyerius (Welmanius) welmani (Stiles et Goldberger, 1910) dans l'estomac de Cervicapra bohor Rüppell 1842, à Benguela (Angola).

Carmyerius bubalis (Innes, 1912) dans l'estomac de Bubalis sp. (Hartebeest) en Rhodésie.

Carmyerius exoporus Maplestone, 1923, dans l'estomac de Tragelaphus spekii Sclat. au Nyassaland.

Carmyerius dollfusi Golvan, Chabaud et Grétillat, 1957, dans le rumen de Bos indicus L. à Madagascar.

Nous n'avons pas l'intention de discuter ici la valeur de chacune des espèces du genre Carmyerius. Pour pouvoir faire un tel travail, il faudrait avoir à sa disposition les différents types et co-types des espèces déjà décrites. Näsmark, en 1937, avait réuni le matériel 
nécessaire à cette étude, mais, dans la bibliographie, nous ne trouvons aucune trace de ses conclusions.

Cependant, l'espèce que nous venons de décrire présente certains caractères permettant de la placer difficilement dans la classification admise actuellement, et nous examinerons rapidement les différents travaux des auteurs ayant étudié la question depuis le travail de Fischoeder en 1903.

En 1910, Stiles et Goldberger, en créant la famille des Gastrothylacidæ et en établissant les différences anatomiques existant entre les trois genres Gastrothylax, Fischoederius et Carmyerius, met-

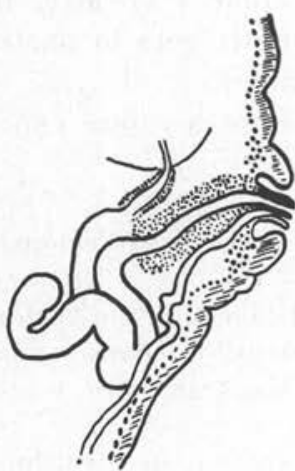

A

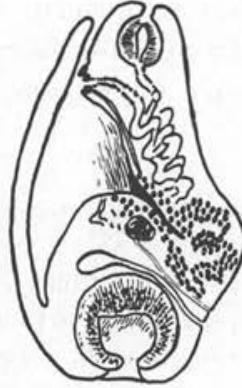

B

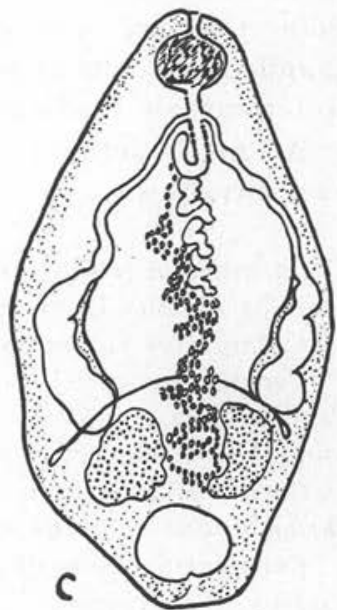

Fig. 8. - C. minutus (Fischoeder, 1901). A. Pore génital ; B. Coupe histologique longitudinale passant par le pore génital; C. Vue de face. (D'après Fischoeder, 1903).

tent de l'ordre dans ce groupe. Il semble cependant admis à l'heure actuelle que leur genre Welmanius, créé pour leur espèce W. welmani, n'est autre qu'un Carmyerius. Les caractères différentiels que donnent ces auteurs, et qui sont basés sur la disposition des anses utérines, du canal déférent et de la vésicule séminale, ne semblent pas motiver la création d'un genre.

La systématique du groupe est claire jusqu'en 1923 où Maplestone publie sa révision sur les Amphistomes. Après avoir revu et étudié les espèces déjà décrites, il met en synonymie avec $C$. spatiosus : C. synethes, C. minutus, C. mancupatus, C. bubalis et $C$. welmani. Par contre, il admet la valeur de l'espèce $C$. cruciformis, décrite pourtant à partir de formes immatures, où la glande de Mehlis et les vitellogènes n'étaient pas encore formés. 
Pour l'établissement de ces synonymies, il considère que l'existence ou l'absence de certains caractères anatomiques sont le résultat d'accidents de rétraction du matériel dans le milieu conservateur. Il passe sous silence tous les détails histologiques dont s'est servi Fischoeder dans la description de ses espèces. Il est vrai que Maplestone étudiait son matériel in toto, après éclaircissement, ou sur coupes faites « au rasoir », alors que Fischoeder travaillait sur coupes histologiques sériées.

L. Travassos, en 1934, admet l'opinion de Maplestone ; par contre, Ben Dawes, en 1936, prétend que cet auteur s'est un peu trop hâté de conclure, et qu'il n'est pas d'espèce plus valable que le $C$. minutus de Fischoeder. Au sujet de C. spatiosus, il le considère comme une espèce distincte et pense qu'il y a lieu de grouper sous le terme de $C$. synethes les espèces : $C$. mancupatus, $C$. minutus, $C$. bubalis et $C$. welmani, qui sont peut-être différentes, mais qui demandent une étude ultérieure.

Näsmark, en 1937, pense lui aussi que Maplestone a rassemblé sous le nom de C. spatiosus des espèces qui doivent être différentes.

Si l'on consulte le gros travail qu'a fait Fischoeder en 1901, 1902, 1903 et 1904 sur les Amphistomata, on est bien obligé d'admettre que $C$. mancupatus, $C$. synethes et $C$. minutus sont des espèces distinctes. En ce qui concerne $C$. synethes en particulier, il présente un atrium génital tellement développé qu'une pareille disposition du pore génital ne se retrouve que chez Bothriophoron bothriophoron (Bräun, 1892) Stiles et Goldberger, 1910. Les détails et les figures que donne l'auteur sont tellement précis, qu'en toute bonne foi, on doit accepter la valeur des trois espèces précédentes. En conséquence, nous nous référons aux publications de Fischoeder pour la discussion de l'espèce qui nous intéresse, en les considérant comme un travail de base dans l'étude des Gastrothylacidx.

La taille très réduite du Carmyerius que nous venons d'étudier le rapproche de $C$. minutus, mais cette dernière espèce a un pore génital sans atrium génital développé et à musculature moyenne, alors que, chez nos spécimens, le même organe est très fortement musclé et placé au fond d'une cavité dont l'orifice est fermé par un sphineter.

Comme autres différences avec $C$. minutus, nos exemplaires ont des testicules volumineux ( 1.500 à $1.700 \mu)$, non lobés, accolés contre les parois latérales du corps et ne faisant pas saillie dans le milieu du plancher de la poche centrale, alors que, chez C. minutus, les testicules sont lobés, de taille moyenne (700 à $800 \mu)$, éloignés de la paroi du corps et font saillie dans la poche centrale. De plus, sur 
nos exemplaires, les glandes vitellogènes sont dorso-latéro-ventrales les culs-de-sac cæcaux arrivent à peine au pôle supérieur des testicules, la cavité acétabulaire est très peu profonde, alors que, chez C. minutus, les vitellogènes sont ventrales, les cæca se terminent aux environs de la demi-hauteur des testicules, et l'acétabulum a une cavité très profonde (fig. $\mathrm{n}^{\circ} 8, \mathrm{~A}, \mathrm{~B}$ et $\mathrm{C}$ ).

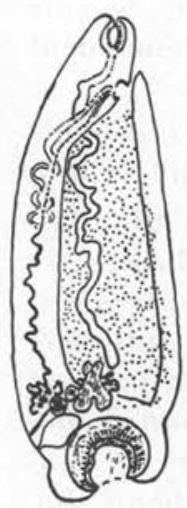

A
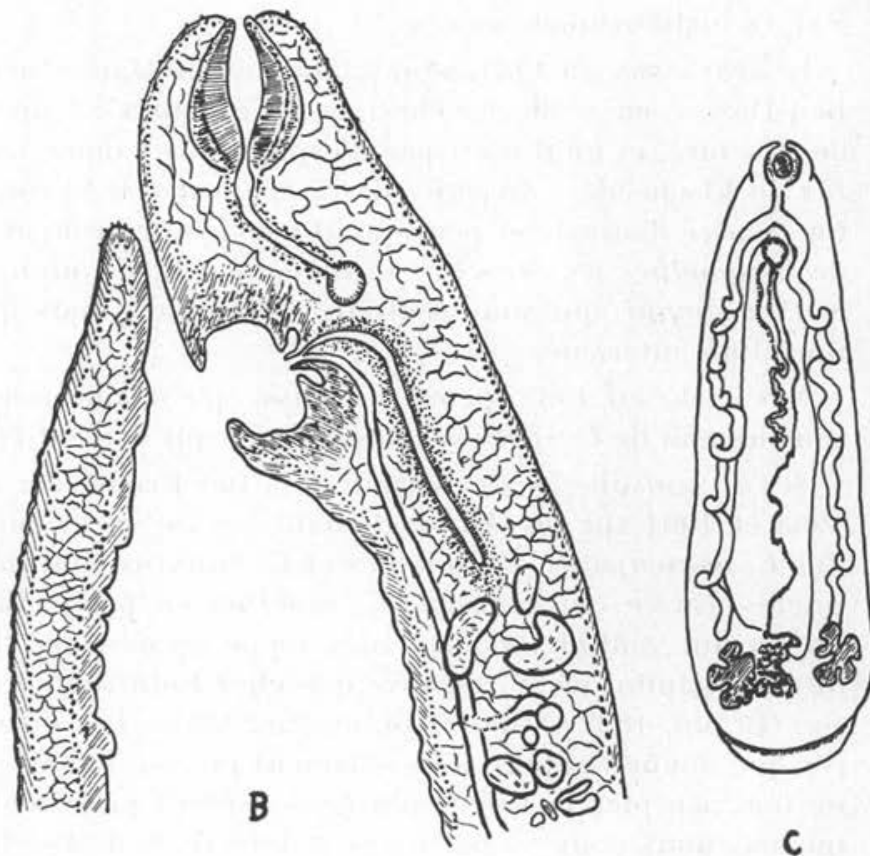

FIG. 9. - C. synethes (Fischoeder, 1901). A. Coupe histologique longitudinale passant par le pore génital ; B. Coupe histologique du pore génital et de la ventouse orale; C. Vue de face. (D'après Fischoeder, 1903).

Parmi toutes les autres espèces déjè décrites, nous ne pouvons comparer nos exemplaires qu'à $C$. synethes, qui est la seule à présenter un atrium génital spacieux pourvu d'une musculature aussi puissante que celle que nous avons remarquée chez le Carmyerius que nous venons de décrire. Laissant de côté l'épaisseur des parois musculaires et la profondeur de cette cavité génitale, ainsi que la longueur de la papille, caractères toujours sujets à controverse, nous nous bornerons à énumérer les différences trouvées entre $C$. synethes et le matériel que nous avons étudié.

Chez $C$. synethes (fig. $\mathrm{n}^{\circ} 9, \mathrm{~A}, \mathrm{~B}$ et $\mathrm{C}$ ), l'œsophage est aussi long 
que la ventouse orale qui est ovalaire, la ventouse postérieure présente une cavité acétabulaire profonde, l'orifice de la poche centrale est très éloigné de la bouche $(1 \mathrm{~mm}$.), la prostate est très longue $(1.200$ à $1.400 \mu)$, les glandes vitellogènes sont latérales et un peu dorsales et les testicules qui sont fortement lobés ont des dimensions moyennes (800 à $1.000 \mu$ ).

Par contre, notre Carmyerius a un œsophage beaucoup plus court que la ventouse orale qui est sphérique, l'orifice de sa poche centrale est beaucoup plus proche de la bouche $(360 \mu)$, la pars prostatica est courte $(500$ à $600 \mu)$, et nous venons de voir que les testicules ne présentaient pratiquement pas de lobulation, que les vitellogènes étaient réparties dans toutes les parois du corps, et que la cavité acétabulaire était très faible.

Nous considérons l'espèce que nous venons de décrire comme nouvelle et la dédions à notre confrère, le $\mathrm{D}^{r}$ Graber, qui a eu l'amabilité de nous envoyer un matériel aussi intéressant.

Types et paratypes: Dans les collections de l'Institut de Parasitologie de la Faculté de Médecine de Paris.

Paratypes: Dans les collections du Laboratoire Central de l'Elevage à Dakar, et dans les collections de l'auteur.

Hôtes : Bos taurus L. et Ovis aries L.

Localisation: Rumen.

Provenance: Abattoirs de Fort-Lamy (République du Tchad).

D'après l'étude des publications traitant de la systématique du genre Carmyerius, nous pensons pouvoir donner ci-dessous une clé pour la détermination des espèces de ce genre.

\section{Clé de détermination des espèces du genre Carmyerius}

Remarque. - La plupart des caractères utilisés dans cette clé doivent être recherchés sur coupes histologiques sériées, seul moyen d'étude valable pour la détermination de ces Trématodes.

1. - Culs-de-sac cæcaux dépassant la partie moyenne du corps 3

2. - Culs-de-sac cæcaux atteignant seulement la partie moyenne

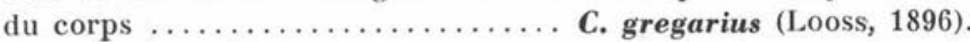

3. - Pore génital s'ouvrant dans la poche centrale .......... 5

4. - Pore génital placé en dehors de la poche centrale .........

C. exoporus Maplestone, 1923. 
5. - Pore génital avec musculature plus ou moins forte mais ne présentant pas d'atrium génital profond circonscrit par une

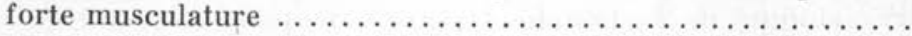

6. - Pore génital avec forte musculature et présentant un atrium génital profond circonscrit par une forte musculature ......

7. - Espèce de grande taille (7 à $11 \mathrm{~mm}$.), œsophage aussi long que la ventouse orale qui est ovalaire. Orifice de la poche centrale placé à environ $1 \mathrm{~mm}$. de la bouche. Pars prostatica allongée $(1.200$ à $1.400 \mu)$. Vitellogènes latérales et un peu dorsales. Testicules très lobés $(800$ à $1.000 \mu)$. Acétabulum avec cavité profonde ......... C. synethes (Fischoeder, 1901).

8. - Espèce de petite taille ( 3 à $5 \mathrm{~mm}$.). CEsophage plus court que la ventouse orale qui est sphérique ou subsphérique. Orifice de la poche centrale proche de la bouche $(350 \mu)$. Pars prostatica courte $(500$ à $600 \mu)$. Vitellogènes dorso-latéro-ventrales. Testicules volumineux $(1.500$ à $1.700 \mu)$ allongés et non lobés. Acétabulum avec cavité très peu profonde .... C. graberi $\mathrm{n}$. sp.

9. - Culs-de-sac cæcaux atteignant seulement le sommet des testi-

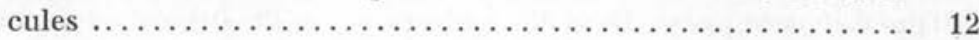

10. - Culs-de-sac cæcaux n'atteignant pas le sommet des testicules

11. - Culs-de-sac cæcaux atteignant la demi-hauteur des testicules

12. - Testicules lobés (rosette). Division cæcale en face du pore génital qui est fortement musclé. Coupe transversale de la poche centrale en forme de croix .. C. cruciformis (Leiper, 1910).

13. - Canal de Laurer et canal excréteur s'ouvrant à l'extérieur par un orifice commun ........... C. wenyoni (Leiper, 1908).

14. - Canal de Laurer et canal excréteur s'ouvrant à l'extérieur par deux orifices distincts ........... spatosius (Brandes, 1898).

15. - Division cæcale au-dessous de l'orifice génital et glandes

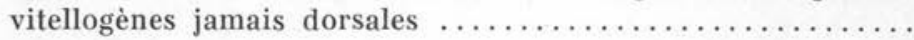

16. - Division cæcale au niveau de l'orifice génital et glandes vitellogènes distribuées dans toutes les parois du corps ....

17. - Espèce de petite taille (3 à $7 \mathrm{~mm}$.). Pore génital avec musculature forte. Testicules lobés. Prostate courte, 400 à $500 \mu \ldots$

C. minutus (Fischoeder, 1901).

18. - Espèce de grande taille (7 à $11 \mathrm{~mm}$.). Pore génital avec musculature faible. Testicules non lobés. Prostate longue, $1.500 \mu$ $\ldots \ldots \ldots \ldots \ldots \ldots$. dollfusi Golvan, Chabaud et Gretillat, 1957.

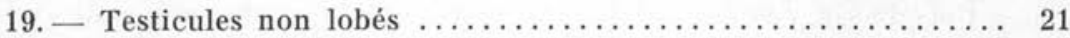

20. - Testicules lobés ............ we wani St. et Gold., 1910.

21. - Prostate longue. Esophage long

C. mancupatus (Fischoeder, 1902).

22. - Prostate courte ? Esophage court ? ... C. bubalis (Innes, 1912). 
En ce qui concerne, $C$. bubalis, la description de cette espèce est tellement confuse et incomplète (aucune mensuration des différents organes) qu'il nous est difficile de la placer dans cette clé.

D'après les figures données par Innès en 1912, et qui sont surtout schématiques, C. bubalis serait très proche de C. mancupatus : poche centrale à section triangulaire, testicules arrondis non lobés, disposition identique des vitellogènes, de l'ovaire, de la glande de Mehlis et de la vésicule excrétrice.

\section{RÉSUMÉ}

Un lot important d'Amphistomes prélevés dans les réservoirs gastriques de Bovins, Ovins et Caprins abattus à Ati, Abecher, Bongor et Fort-Lamy (République du Tchad), nous a permis d'identifier les espèces suivantes :

Paramphistomum microbothrium Fischoeder, 1901.

Calicophoron calicophorum (Fischoeder, 1901) Näsmark 1957.

Stephanopharynx compactus (Fischoeder, 1901).

Carmyerius spatiosus (Brandes, 1898).

Nous pouvons, en outre, confirmer l'existence en Afrique de l'espèce Bothriophoron bothriophoron (Bräun, 1892) Stiles et Goldberger, 1910, décrite de Madagascar, et seulement trouvée à quelques exemplaires par Dinnik, en 1950, aux abattoirs de Nairobi (Kénya), dans un mouton et une chèvre.

Parmi le matériel examiné, nous avons eu la chance de trouver un Carmyerius présentant des caractères intermédiaires entre C. minutus et $C$. synethes, et que nous considérons comme nouveau. Nous le décrivons sous le nom de $C$. graberi n. sp. Nous faisons suivre cette description d'une clé de détermination des différentes espèces de Carmyerius connues et décrites à l'heure actuelle.

\section{Laboratoire Central de l'Elevage «Georges-Curasson », Dakar}

(Directeur : P. MORNET)

Nous remercions M. le Professeur R.-P. Dollfus, du Muséum National d'Histoire Naturelle de Paris, des conseils qu'il nous a donnés pour ce travail.

D'autre part, c'est grâce à l'obligeance de M. le Professeur A.-G. Chabaud, de l'Institut de Parasitologie de la Faculté de Médecine de Paris, 
et du Docteur Watson, Directeur de la revue Helmintological Abstracts, de Londres, que nous avons pu nous procurer les photocopies de certains articles difficiles à consulter. Nous les en remercions bien vivement.

\section{BibLiOgRAPHIE}

Brandes (G.), 1898. - Die Gattung Gastrothylax. Naturfosh. Gesellsch. z. Halle, XXI, 195-225, pl. VII, fig. 1-16, et pl. VIII, fig. 1-16.

Dawes (Ben), 1936. - On a collection of Paramphistomida from Malaya with revision of the genera Paramphistomum Fischoeder, 1901 and Gastrothylax Poirier, 1883. Parasitology, XXVIII (3), 330-354, fig. 1-7.

Fischoeder (F.), 1901. - Die Paramphistomiden der Säugetiere. Zoolog. Anzeig., XXIV, $\mathrm{n}^{\circ} 646,367-375$.

- 1902. - Die Paramphistomida der Säugetiere. Inaug. Dissert. Königsberg, 59 pp., 4 fig.

- 1903. - Die Paramphistomiden der Säugetiere. Zoolog. Jahrbücher, XVII, (4), 485-660, pl. I-105.

- 1904. - Beschreibung dreier Paramphistomiden der Säugetieren. Zoolog. Jahrbücher System., XX, (5), 453-470, fig. A-C, et pl. XV-XVII, 1-11.

- 1904. - Weitere Mitteilungen über Paramphistomiden der Säugetiere. Centralbl. f. Bakt. Parasitenk. u. Infektionskr., I Abt., Orig., XXXV, (5), $598,601$.

Fukur (T.), 1929. - Studies on Japanese Amphistomatous parasites with revision of the group. Japanese Jl. Zoolog., II, (3), 219-351.

Golvan (Y.), Chabaud (A.-G.) et Grétillat (S.), 1957. - Carmyerius dollfusi n. sp. (Trematoda, Gastrothylacidæ), parasite des bovidés à Madagascar. Ann. Parasit. hum. comp., XXXI, (1-2), 56-70, fig. 1-9.

Grétillat (S.), 1958. - Maintien du genre Bothriophoron Stiles et Goldberger. 1910, et valeur de l'espèce Paramphistomum Bothriophoron (Braun, 1892), Fischoeder, 1901 (Trematoda, Paramphistomatida), parasite du réticulum du zébu malgache. Ann. Parasit. hum. comp., XXXIII, (3), 240-253, fig. 1-7.

INNES (J. A.), 1912. - Gastrothylax bubalis n. sp., with a few notes on the genus Gastrothylax (Poirier). Parasitology, V, 217-225.

LeIPER (R. T.), 1908. - An account of some Helminths contained in Dr C. M. Wenyon's collection from the Sudan. III d. Rep. Wellc. Res. Laborat. Khartoum, 187-199.

- 1910. - The Entozoa of the Hippopotamus. Proceed. Zoolog. Soc. London, I, 233-251.

Looss (A.), 1896. - Recherches sur la faune parasitaire de l'Egypte. Mém. Inst. Egypte, III, 1-252.

Maplestone (P.-A.), 1923. - A revision of the Amphistomata of Mammals. Ann. Trop. Méd. Parasit., XVII, (2), 113-212, fig. text., 1-32, et pl. V-VIII.

NÂsmark (K. E.), 1937. - A revision of the trematode family Paramphistomatider. Inaug. Dissert. Uppsala. Zoologisk. Bridrag. Fran. Uppsala, XVI, 301-566, fig. text., 1-104, et pl. I-XIII.

Porrier (J.), 1883. -- Description d'Helminthes nouveaux du Palonia frontalis. Bull. Soc. Philomat., 73-80 ; pl. II, fig. 1-32. 
SkrJabin (C. I.), 1949. - Trématodes des animaux sauvages et domestiques (en russe). Akad. Nauk. CCCP., t. III, 623 pages, fig. text., 1-145, et pl. I-V).

Stiles (Ch. W.) and Goldberger (J.), 1910. - A study of the anatomy of Watsonius n. g. watsoni of man and of 19 allied species of mammalian Trematode Worms of the superfamily Paramphistomoidea. Treas. Dept. Pub. Health. Marine Hosp. Serv. U.S. Hyg. Laborat. Bull., ${ }^{\circ}$ 60, U.S.A., 264 pages, fig. 1-205.

Stunkard (H. W.), 1929. - The parasitic worms collected by the American Museum Natural History (Expedition to the Belgian Congo). Bull. Amér. Mus. Nat. Hist., LVIII, 233-289, fig. 1-37.

Travassos (L.), 1934. - Synopse dos Paramphistomoidea. Mem. Inst. Oswaldo Cruz, XXIX, 19-170, fig. text., 180, pl. I-III.

\section{LEGENDE DES ABREVIATIONS}

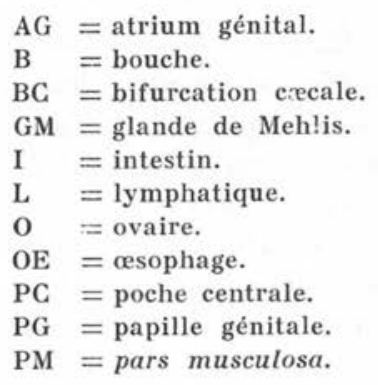

$\mathrm{PP}=$ pars prostatica.

$\mathrm{SA}=$ sphincter atrial.

$\mathrm{SP}=$ sphincter papillaire

$\mathrm{T}=$ testicule.

$\mathrm{U}=$ utérus.

$\mathrm{V}=$ vitellogènes.

$\mathrm{VE}=$ vésicule excrétrice.

vo $=$ ventouse orale

$\mathrm{VP}=$ ventouse postérieure.

vS = vésicule séminale . 